\title{
Surgical Options for Malignant Skin Tumors of the Hand
}

\author{
Min Ji Yun ${ }^{1}$, Ji Ung Park ${ }^{2}$, Sung Tack Kwon ${ }^{1}$ \\ ${ }^{1}$ Department of Plastic and Reconstructive Surgery, Seoul National University College of Medicine, Seoul; ${ }^{2}$ Department of Plastic and \\ Reconstructive Surgery, SMG-SNU Boramae Medical Center, Seoul National University College of Medicine, Seoul, Korea
}

Background Primary malignant tumors of the hand, although unusual, may present varied and often complex clinical problems. The main treatment modality of skin cancer of the hand has changed.

Methods We retrospectively reviewed the medical records of 43 patients who underwent surgery for malignant skin tumors of the hand during an 18-year period, from September 1994 to February 2012. The characteristics of the tumor, methods of reconstruction, and long-term results were reviewed.

Results We had 43 patients with 27 melanomas, 14 squamous cell carcinomas, and 2 sarcomas. Their ages ranged from 19 to 74 years (mean, $53.4 \pm 14.5$ years), from 46 to 79 years (mean, $59.7 \pm 9.6$ years), and from 15 to 43 years (mean, $29 \pm 19.8$ years), respectively. Thirtyfour cases occurred on the fingertip (16 of those cases on the thumb), 5 cases occurred on the palm, and 4 cases on the dorsum of the hand. Amputation was most frequently used in early cases, but recently, tissue-sparing excision has been performed frequently. The incidence of local recurrence was 3 cases and distant metastasis was 1 case, and the 5-year survival rate was 100\%, except in 4 cases due to follow-up loss.

Conclusions The principles of treatment-to be curative and to preserve function and appearance-are important points. "Preservative surgery" preserves function and cosmesis of the involved finger or hand dorsum or palm. Preservative surgery not only emphasizes less resection and surgery of a smaller scale, but also optimal reconstruction of the soft tissue defect of the digit.

Keywords Skin neoplasms / Melanoma / Carcinoma, squamous cell / Surgical flaps
Correspondence: Sung Tack Kwon Department of Plastic and Reconstructive Surgery, Seoul National University College of Medicine, 101 Daehak-ro, Jongno-gu, Seoul 110-744, Korea Tel: $+82-2-760-3759$ Fax: +82-2-745-5986 E-mail: stk59@snu.ac.kr
This article was presented at the 69th Congress of the Korean Society of Plastic and Reconstructive Surgeons on November 11-13, 2011, in Seoul, Korea.

No potential conflict of interest relevant to this article was reported.

\section{INTRODUCTION}

The hands represent only $1 \%$ to $2 \%$ of the total body surface area, while skin tumors affecting the hands account for $10 \%$ to $15 \%$ of all skin malignancies [1]. Primary malignant skin tumors of the hand may present varied and often complex clinical prob- lems. Moreover, managing tumors at this site demands special consideration, as the skin of the hand is specialized and structurally unique, balancing the need for fine sensibility, mobility for complex motor skills, and durability to withstand wear and tear. The dorsal subcutaneous layer is thin but must protect the deep structures while allowing their functioning. 
The main treatment modality of skin cancer of the hand has changed through the decades. In the past, the mainstream treatment was radical excision, including ray amputation regardless of the extent or stage of cancer. Recently, in cases of noninvasive or low grade skin cancer, proper excision of the skin cancer and suitable reconstruction of the soft tissue defect has been preferred due to regard for functional and aesthetical preservation [2].

Here, we reviewed 43 primary skin malignancies of the hand seen at the Department of Plastic and Reconstructive Surgery of our medical institution during the past 18 years. The cell type, tumor extent, method of reconstruction, and long-term results of each case were compared to those of previous studies to uncover any findings that may suggest some helpful advances for the treatment of these specific types of cases.

\section{METHODS}

A retrospective review of 43 cases who underwent surgery for malignant skin tumors of the hand during an 18-year period from September 1994 to February 2012 was conducted. This study was approved by the Institutional Review Board of Seoul National University Hospital (H-1301-070-459). The inclusion criteria comprised primary skin malignancies, except premalignant lesions distal to the distal wrist crease. Patient files were reviewed to document demographics and identify details, including the histopathology, extent of excision, and reconstruction technique. The outcome parameters included local recurrence, metastasis, and survival. Clinic visits, as well as telephone interviews, revealed the disease free states of 39 patients up to the present; however, the other 4 patients treated for malignant melanoma could not be reached. The follow-up period of the patients lost to follow up was 6 months for 2 patients, 12 months for 1 patient, and 3 years for 1 patient.

\section{Table 1. Demographic data}

\begin{tabular}{|lc|}
\hline Variable & Data \\
\hline Mean age (yr) & \\
Male & $53.6 \pm 16.2$ \\
Female & $55.2 \pm 12.4$ \\
Age range (yr) & $15-79$ \\
Age distribution (yr) & \\
$<30$ & 2 \\
$31-40$ & 5 \\
$41-50$ & 6 \\
$51-60$ & 15 \\
$61-70$ & 9 \\
$71-80$ & 6 \\
Male:Female & $1: 0.87$ \\
\hline
\end{tabular}

\section{RESULTS}

The average age of the patients was $55.6 \pm 14.6$ years (range, 15 to 79 years); 23 were male and 20 were female. The mean follow-up period of clinic visits was 48 months (range, 11 to 201 months) (Table 1). A total of 27 patients were diagnosed with malignant melanoma, while 14 had squamous cell carcinoma, and 2 had other sarcomas (epithelioid sarcoma, dermatofibrosarcoma) (Table 2). By histologic subtype, 14 cases were melanoma in situ, and 13 cases were invasive melanoma. Of these, 4 patients were in stage $\mathrm{T} 1$ ( $\leq 1.00 \mathrm{~mm}$ thick), 3 patients were in stage T2 (1.01 to $2.00 \mathrm{~mm}$ thick), 5 patients were in stage T3 (2.01 to $4.00 \mathrm{~mm}$ thick), and one was in stage $\mathrm{T} 4$ (> $>4.00 \mathrm{~mm}$ thick). One case was squamous cell carcinoma in situ, and the other 13 cases were invasive squamous cell carcinoma. Thirtyfour lesions presented at the finger-tip: 16 cases on the thumb, 18 cases on the other fingers, 4 cases on the dorsum of the hand, and 5 cases on the palm. In the malignant melanoma patients, 13 lesions presented on the thumb and 13 cases on the other finger tips, with 1 case on dorsum of the hand. In the squamous cell carcinoma patients, 3 lesions presented on the thumb, 5 cases on the other fingers, 3 cases on the palm, and 3 cases on the dorsum. Malignant melanomas tend to occur as fingertip lesions, whereas squamous cell carcinoma occur everywhere on the hand. Epithelioid sarcoma occurs as palmar lesions, and dermatofibrosarcoma on the dorsal side of the hand.

The surgical margins were differentiated based on the tumor type and location under our treatment principles. Surgical excision was performed with a safety margin of $3 \mathrm{~mm}$ for the squamous cell carcinoma and $5 \mathrm{~mm}$ for the malignant melanoma in most cases. Excision was done to the subcutaneous level. In the fingertip cases, the entire nail complexes were removed en bloc

Table 2 . Incidence and demographics by malignancy type

\begin{tabular}{|lccc|}
\hline Variable & $\begin{array}{c}\text { Malignant } \\
\text { melanoma }\end{array}$ & $\begin{array}{c}\text { Squamous cell } \\
\text { carcinoma }\end{array}$ & Sarcoma \\
\hline No. of patients & 27 & 14 & 2 \\
Histologic subtype & 14 & $1\left(1^{\text {a) }}\right)$ & 0 \\
In situ & $13\left(1^{\text {a) }}\right)$ & $13\left(1^{\text {al)}}\right)$ & 2 \\
Invasive & & & 0 \\
Location & 13 & 3 & 0 \\
Thumb & 13 & 6 & 1 \\
Other finger & 1 & 3 & 1 \\
Palm & 0 & 2 & $29 \pm 19.8$ \\
Dorsum & & & $15-43$ \\
Age (yr) & $53.4 \pm 14.5$ & $59.7 \pm 9.6$ & $1: 0$ \\
Mean & $19-74$ & $46-79$ & \\
Range & $1: 1.25$ & $1: 0.55$ & \\
Male:Female & & & \\
\hline a) Local recurrence case. & & & \\
\hline
\end{tabular}


as one unit with subperiosteal dissection. If secondary change occurred or clinically invaded the periosteum, bone resection was performed. The surgical margin was $20 \mathrm{~mm}$ in epithelioid sarcoma, and $30 \mathrm{~mm}$ in dermatofibrosarcoma.

All of the lesions were completely excised according to the histopathologic findings. For malignant melanoma, amputations were the most frequently used excision and reconstructive method, followed by skin graft, venous free flap, and free flap. There was no case of amputation among the squamous cell carcinoma and sarcoma patients. Two patients with palpable lymph nodes underwent axillary lymph node dissection in early cases.

Reconstructive methods for soft tissue defects are summa-

Table 3. Defect reconstruction by malignancy type and location

\begin{tabular}{|lcccc|}
\hline Malignancy type & Thumb & Finger tip & Palm & Dorsum \\
\hline Malignant melanoma & & & & \\
Skin graft/Local flap & 3 & 4 & 1 & - \\
Amputation & 6 & 7 & - & - \\
Free flap (venous free flap ${ }^{\text {al) }}$ & $4(4)$ & $2(2)$ & - & - \\
Squamous cell carcinoma & & & & \\
Skin graft/Local flap & - & 3 & 3 & 2 \\
Amputation & - & - & - & - \\
Free flap (venous free flapa) & $3(3)$ & $2(2)$ & - & $1(1)$ \\
Sarcoma & & & & - \\
Skin graft/Local flap & - & - & 1 & - \\
Amputation & - & - & - & - \\
Free flap (venous free flap ${ }^{\text {al) }}$ & - & - & $1(0)$ & - \\
\hline a)Cases of venous free flap. & & & & \\
\hline
\end{tabular}

rized in Table 3. Before choosing the method of reconstruction for the skin defects of the hand, the patients received detailed information about all options. Reconstructive strategies were determined by the location of the lesion and malignancy grade, as well as the patient's choice. Skin graft or local flap (Fig. 1) was most often indicated in low grade malignancy, squamous cell carcinoma, and dorsum or palm lesions. Amputation (Fig. 2) was most common in malignant melanoma on the fingertip, including the thumb, but these patients have not experienced recurrence. Therefore, since 2009, tissue-sparing excision and reconstruction with venous free flap (Fig. 3) was more frequently performed for finger tip lesions, especially on the thumb.

\section{Fig. 2. A case of finger tip amputation}

(A) A malignant melanoma on the nail complex of the left thumb with secondary change in a 64-year-old woman. (B) Amputation was performed at the distal interphalangeal joint level.
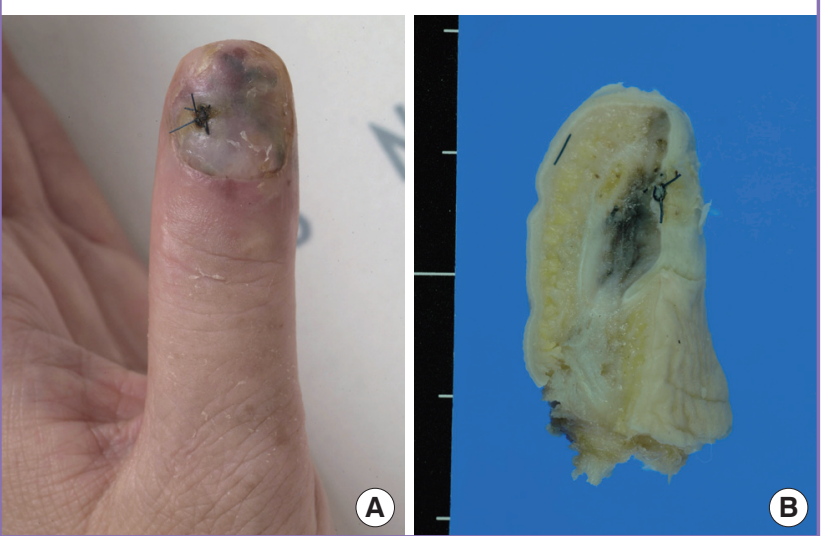

Fig. 1. A case of reconstruction with skin graft

(A) A $2 \mathrm{~cm} \times 2 \mathrm{~cm}$ squamous cell carcinoma lesion on the right palm in a 54-year-old man. (B) The tumor was excised with a $5 \mathrm{~mm}$ resection margin and reconstruction with split thickness skin graft. (C) Twelve months postoperatively, the grafted skin was well taken without local recurrence.
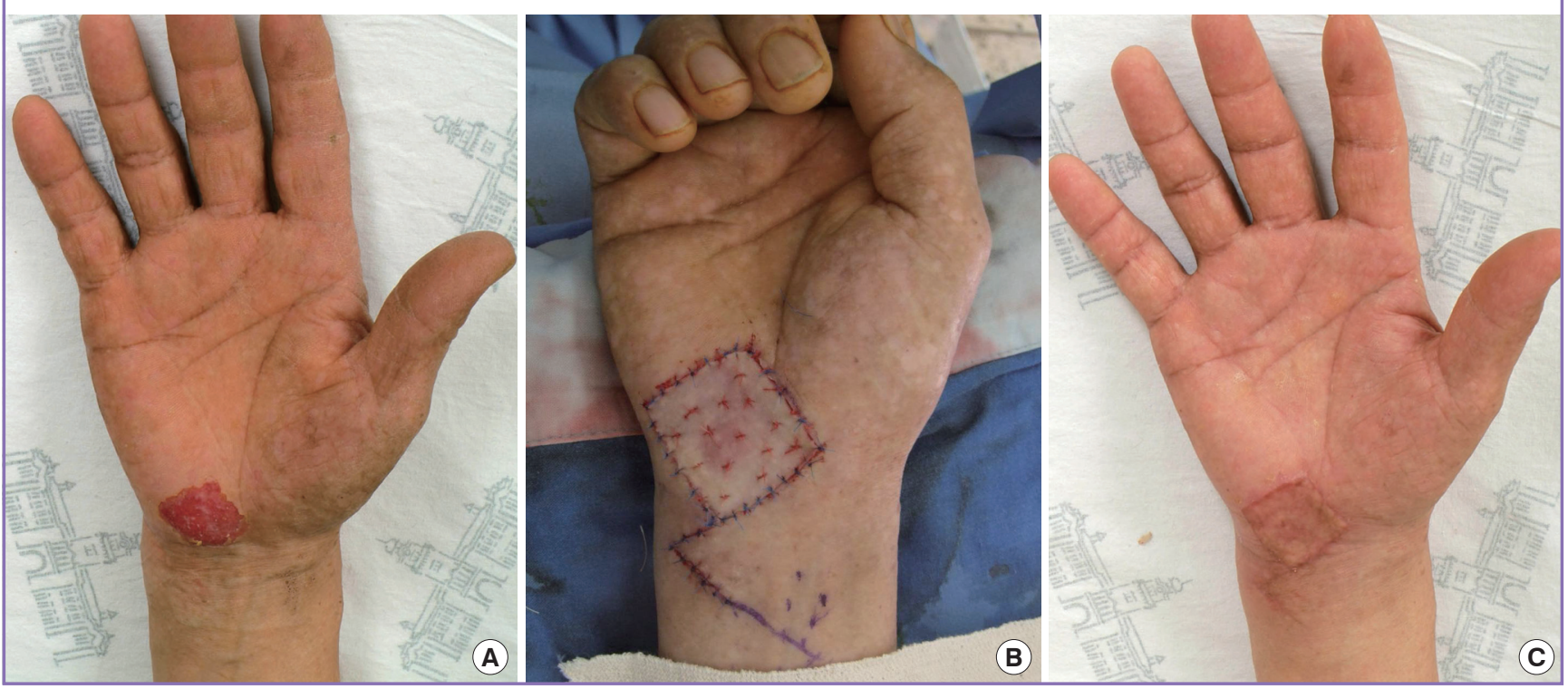
Fig. 3. A case of reconstruction with venous free flap

(A) A $5 \mathrm{~cm} \times 2.5 \mathrm{~cm}$ squamous cell carcinoma on the dorsum of the proximal phalanx in the left middle finger. (B) The tumor was excised to the upper layer of the tendon sheath, including the whole skin territory of the proximal phalanx. (C) A $5 \mathrm{~cm} \times$ $7 \mathrm{~cm}$ venous flap from the ipsilateral volar side of the distal forearm. (D) Postoperative 19 months, the flap shows a natural contour of the dorsal finger and web space with similar skin color and texture.
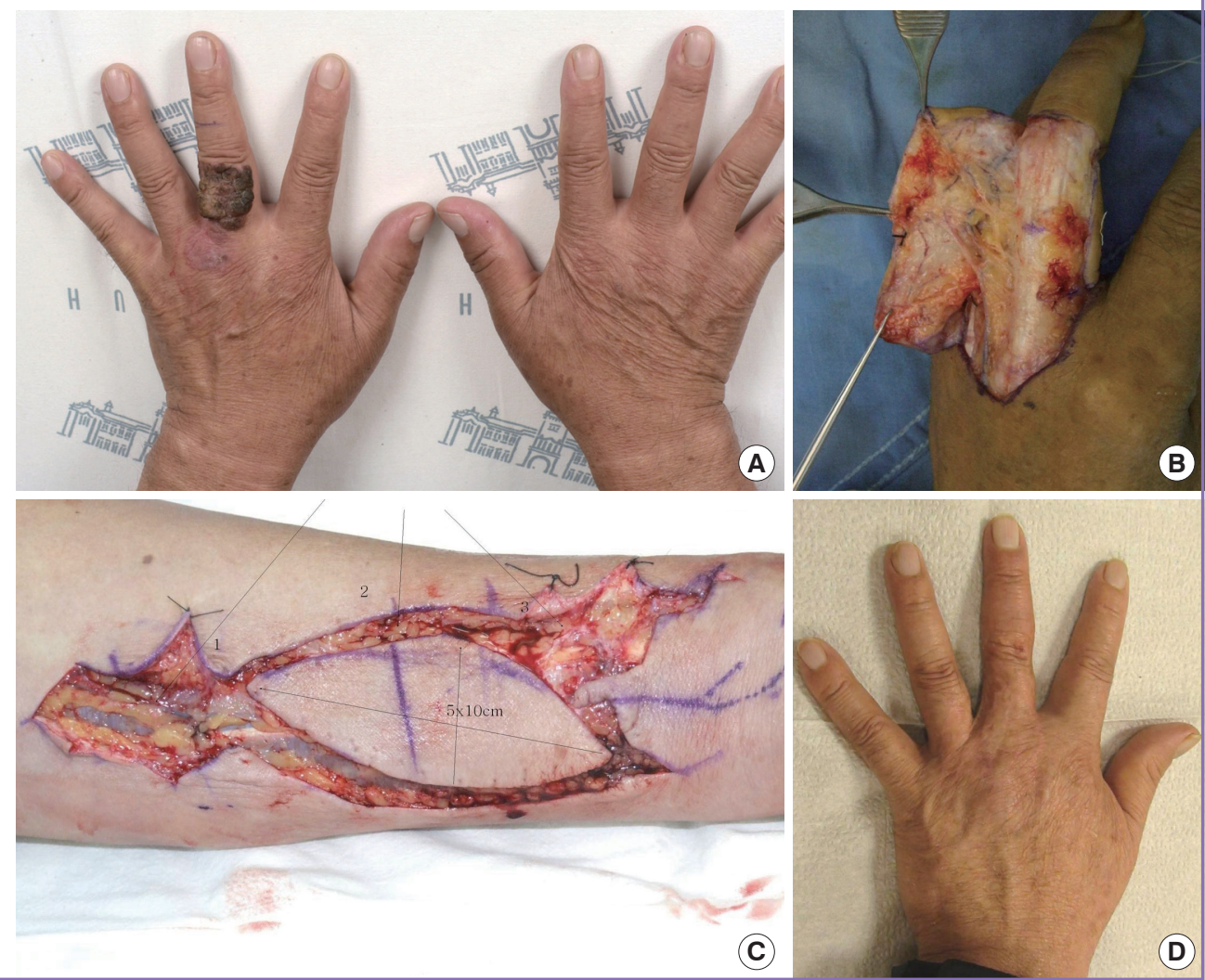

Local recurrence was diagnosed in patients presenting with squamous cell carcinoma $(\mathrm{n}=2)$ and malignant melanoma $(\mathrm{n}=1)$. All of the patients with local recurrence underwent further excision. In the first case, the tumor on the dorsum of the hand was removed with a safety margin of at least $5 \mathrm{~mm}$ and histologically diagnosed squamous cell carcinoma from a burn scar. Fourteen months after the surgery for the primary tumor, a local recurrence was found that was excised with a 5-mm safety margin. There was no further recurrence within an 84-month follow-up period. In the second patient, the tumor on the palm of the hand was removed with a safety margin of at least $5 \mathrm{~mm}$ and histologically diagnosed as squamous cell carcinoma in situ. Eleven months after the surgery, a local recurrence was found that was excised with a 5-mm safety margin. There was no further recurrence within a 22-month follow-up period. In the third patient, the tumor on the right thumb was removed with a safety margin of at least $10 \mathrm{~mm}$ and histologically diagnosed as malignant melanoma (1 mm depth invasion). Nineteen months after surgery of the primary tumor, a local recurrence was found that was amputated to one third of the proximal phalangeal level. This patient developed axillary nodal and lung metastasis after about 4 years, and chemotherapy and radiotherapy were performed. She is still alive within a 43-month follow-up period. There has been no death with an average of 48 months follow up, except four patients who were lost to follow-up, but none of them was regarded to be expired given that their national identification numbers remain valid.

\section{DISCUSSION}

The demographics of our patients are similar to the referral patterns of previous studies. We did not have basal cell carcinoma cases, which may be underreported or not delivered to our hospital because they were treated with methods such as simple excision or chemosurgery by a general practitioner or dermatologist. One consistency among all reports of melanoma of the hand is the paucity of cutaneous primaries on the palm [3-5]; we had only one on the palm of 27 melanoma cases.

Melanomas of the extremities have been known to progress more aggressively. However, only one of the 27 cases of melanoma of the hand recurred and presented distant metastasis. This is best explained by the functional unit of the fingertip lesion. The fingertip is characterized by its dorsal subcutis: layers are very thin but multilayered, which probably provides a draining barrier with scant lymphatics and no hair growth. The multilayer nail complex may act as a draining barrier and contribute to the diminished local recurrence or metastasis rate unless the periosteum underneath is invaded. As such, early identification and 
definitive treatment provide the best opportunity to prevent metastasis. According to Glat et al. [6], in melanoma on the finger tip lesion, this is likely due to delayed presentation, and therefore, higher-stage disease at the time of diagnosis and not due to increased aggressiveness of melanoma in these locations.

Skin cancer management in the hand requires consideration of not only oncologic but also reconstructive principles, while considering both functional and aesthetic results to optimize both the likelihood of cure and quality of life. In addition, the occupational and social status of the patient and surgeon's experience should be considered. We have been moving to preservative surgery with preservation of as much function and aesthetics as possible in the treatment of skin cancer in the hand. Wide excision with phalanx amputation carries a cosmetic and functional cost. It is not satisfactory for the patients and has not shown prolongation of disease-free survival, particularly in the early cases [7]. Recent studies have shown that more distal levels of amputation do not compromise survival or recurrence rates. Many previous studies have reported the validity of conservative surgery in noninvasive or low grade skin cancer of the hand $[8,9]$. In other words, the strategy has changed from radical surgery, including ray amputation, to more conservative treatment. Moehrle et al. [2] described 62 patients with subungual melanoma, 31 of whom underwent amputation in or proximal to the distal interphalangeal joint, and the other 31 of whom underwent "functional" surgery with local excision of the tumor and only partial resection of the distal phalanx. The avoidance of amputations was not associated with an impairment of survival or recurrence-free survival. "Functional" surgery distal to the distal interphalangeal joint did not adversely affect the prognosis of patients, but the functional surgery led to superior aesthetic, functional, and psychological results.

We believe that the invasion depth of the tumor is the most powerful prognostic indicator for recurrence rather than the peripheral resection margin in the skin cancer of the digit, as noted previously [10]. Therefore, if a sufficient resection of the deeper margin was performed with the assessment of the free margin, a reduced peripheral safety margin could be required for curative excision and should facilitate superior functional and aesthetic reconstruction. Our management principle has become more conservative, with preservation of oncologic outcomes. Ten cases of 13 amputations have performed until 2009. Since 2009, our surgical principle has become more strictly tissue-sparing excision, which has been performed frequently. The use of tissuesparing Mohs micrographic surgery for a malignant hand skin tumor is an alternative that has the potential benefit of avoiding amputation. However, it is not available in all centers, including our institution [11].
Among the malignant melanoma patients, more specificially, subungual melanoma, 26 cases presented on the fingertip nail complexes, and only 1 case on the dorsum of the hand. Thirteen cases of these patients had tissue-preserving "functional" surgery, based on the functional unit of the fingertip lesion theory. The others $(n=14)$, which displayed presence of ulceration, satellite lesions, and periosteal invasion, were amputated.

Among the squamous cell carcinoma patients, all cases with fingertip lesions were treated with limited excision for the same reason. The other cases, which showed evidence of periosteal invasion, were greater than $20 \mathrm{~mm}$, displayed satellite lesions or ulceration, or were located on the dorsum of the proximal phalanx, were excised with a surgical margin of $5 \mathrm{~mm}$, corresponding to the current British Association of Dermatologists guidelines for the management of squamous cell carcinoma [12]. According to Maciburko et al. [13], with squamous cell carcinoma, the following were associated with a poorer prognosis: site (the skin overlying the dorsum of the proximal phalanges and web spaces), ulceration, macroscopic diameter greater than $20 \mathrm{~mm}$, tumor thickness greater than $4 \mathrm{~mm}$, and invasion to the subcutaneous fat or beyond.

In our group, local excision with a skin graft or local flap coverage is often indicated if the lesion is superficial. When invasion to the periosteum occurred or was suspected, the size was over $20 \mathrm{~mm}$, or satellite lesions were defined, amputation of a digit was the treatment of choice. However, early stage tumors in younger persons can be treated successfully with excision with a close margin and reconstruction with venous free flap. When secondary changes occurred, the excision range was extended one more layer and a 3 to $5 \mathrm{~mm}$ margin retained. Regional node dissection should be performed only if there are palpable nodes at the epitrochlear or axillary level; however, we did not have any indicated cases. The low rate of sentinel lymph node biopsies reflects the fact that this study predates routine use of sentinel lymph node biopsy in prediction of the prognosis.

There were no recurrences or metastases during the follow-up period; however, the short follow-up period and low number of cases are the limitations of this study. There are no published guidelines on the management of malignant skin cancer in the hand. Anecdotal evidence suggests that, as in aesthetically sensitive areas, such as the face, many surgeons are performing limited excision in an attempt to maintain finger length and function [14].

\section{REFERENCES}

1. Mason ML. Carcinoma of the hand. Arch Surg 1929;18: 2107-58. 
2. Moehrle M, Metzger S, Schippert W, et al. "Functional” surgery in subungual melanoma. Dermatol Surg 2003;29:36674.

3. Keyhani A. Comparison of clinical behavior of melanoma of the hands and feet: a study of 283 patients. Cancer 1977;40: 3168-73.

4. Dubrow R, Flannery JT, Liu WL. Time trends in malignant melanoma of the upper limb in Connecticut. Cancer 1991; 68:1854-8.

5. Quinn MJ, Wikramanayake R, Thompson JF, et al. Nonsubungual melanomas of the hand. J Hand Surg Br 1992;17: 433-6.

6. Glat PM, Spector JA, Roses DF, et al. The management of pigmented lesions of the nail bed. Ann Plast Surg 1996;37: 125-34.

7. Clifford RH, Kelly AP Jr. Primary malignant tumors of the hand. Plast Reconstr Surg (1946) 1955;15:227-32.

8. Ilyas EN, Leinberry CF, Ilyas AM. Skin cancers of the hand and upper extremity. J Hand Surg Am 2012;37:171-8.

9. Mullen JT, Feng L, Xing Y, et al. Invasive squamous cell carcinoma of the skin: defining a high-risk group. Ann Surg Oncol 2006; 13:902-9.

10. Balch CM, Murad TM, Soong SJ, et al. A multifactorial analysis of melanoma: prognostic histopathological features comparing Clark's and Breslow's staging methods. Ann Surg 1978;188:732-42.

11. High WA, Quirey RA, Guillen DR, et al. Presentation, histopathologic findings, and clinical outcomes in 7 cases of melanoma in situ of the nail unit. Arch Dermatol 2004;140: 1102-6.

12. Motley R, Kersey P, Lawrence C, et al. Multiprofessional guidelines for the management of the patient with primary cutaneous squamous cell carcinoma. Br J Plast Surg 2003; 56:85-91.

13. Maciburko SJ, Townley WA, Hollowood K, et al. Skin cancers of the hand: a series of 541 malignancies. Plast Reconstr Surg 2012;129:1329-36.

14. Rayatt SS, Dancey AL, Davison PM. Thumb subungual melanoma: is amputation necessary? J Plast Reconstr Aesthet Surg 2007;60:635-8. 